\title{
DIALLEL ANALYSIS INVOLVING MATERNAL AND MATERNAL INTERACTION EFFECTS
}

\author{
PAULINE B. TOPHAM \\ Scottish Horticultural Research Institute, Invergowrie, Dundee
}

Received I8.iii.66

\section{INTRODUCTION}

MATERNAL and reciprocal effects among the progenies of a set of diallel crosses have been examined by Wearden ( 1964$)$, who derived the expected mean squares for three different analyses under two different models, assuming in each case either maternal or reciprocal effects. He did not, however, examine the implications of both effects occurring in the same material. Where the maternal effects of the varieties used in a diallel are important, it seems reasonable to look for an interaction between such effects and the non-recurrent genotype and to see how far it can be isolated. Such a situation arises, for instance, in studying seed and embryo characters and germination in seed resulting from diallel pollinations. A mathematical appraisal of the situation is given here and illustrated by experimental results of seed size in raspberries after a set of diallel crosses.

\section{THE MODEL}

Assuming the presence of a full set of diallel crosses, i.e., $p^{2}$ combinations involving $p$ parents, the model is:

$$
r_{i j}=\mu+g_{i}+g_{j}+m_{i}+s_{i j}+n_{i j}+e_{i j}
$$

where the symbols represent respectively the total for the cross between the $i$ th female and the $j$ th male, the mean, the $i$ th and the $j$ th parental effects, the $i$ th maternal effect, the genic interaction between the $i$ th and the $j$ th parental effects, the interaction between the $i$ th maternal effect and the $j$ th parental effect and an error. The model used is fixed rather than random, since the parents will have been selected to represent a range of behaviour, and the estimation of the various effects for each parent is one of the aims of the experiment. This is an extension of the Sprague and Tatum (1942) technique for estimating general and specific combining ability, but including maternal effects and their interactions, and using a different experimental design $\left(p^{2}\right.$ crosses instead of $\left.\frac{p(p-I)}{2}\right)$.

The restraints to be placed on the effects require some consideration. A diallel table is a special form of the two-way table, with the peculiarity that rows and columns contain the same parents in different roles. The parental and maternal effects are main effects, involving respectively 
the sum and difference of the appropriate row and column totals. They are restrained by $\sum_{1}^{p} g_{i}=0, \sum_{1}^{p} m_{i}=0$, and although summation is over parents, rather than over rows and columns, the two together effectively fix row and column totals. These are primary restraints and each accounts for $p-1$ degrees of freedom.

The other two parameters, $s_{i j}$ and $n_{i j}$ are both components of the first order interaction. Like an ordinary row/column interaction they are both subject to the restraints $\sum_{i=1}^{p} s_{i j}=\sum_{j=1}^{p} s_{i j}=0=\sum_{i=1}^{p} n_{i j}=\sum_{j=1}^{p} n_{i j}$, but these are secondary restraints and do not account for any degrees of freedom. The interaction is being partitioned, and fitting the parameters for genic interaction introduces a condition, $s_{i j}=s_{j i}$, which accounts for the $p(p-1) / 2$ degrees of freedom associated with this term in the diallel analysis.

Whilst there seems no inherent biological reason why the interaction $n_{i j}$ between the $i$ th maternal parent and the $j$ th paternal parent should be equal in size but of opposite sign to $n_{j i}$, the interaction between the $j$ th maternal parent and the $i$ th paternal parent, there is a statistical necessity in that the sum of the reciprocals has been used to estimate the genic interaction and the condition, $n_{i j}+n_{j i}=0$, follows from this as a secondary restraint. Similarly it seems biologically reasonable that a self should have both genic and maternal interactions, that is, that both $s_{i i}$ and $n_{i i}$ should exist; but the biological impossibility of reciprocal replication for the selfs makes it impossible to do more than estimate one interaction parameter for each of them. This may be called genic because this is the first parameter to be fitted and a more fundamental cause of variation, but caution should be exercised in interpreting the values obtained when both genic and maternal interactions are significant.

Using these restraints, the expectations of the mean squares in the Hayman analysis (Hayman, I954) are as shown in table I. Valid F-tests of the usual type are available for all terms, provided that replication allows an estimate of residual error, but the test for genic effects lacks sensitivity since the $(a)$ mean square must be tested against the $(c)$ mean square if this is significant.

\section{MATERNAL v. PATERNAL INHERITANCE}

As Jinks has pointed out (footnote, Wearden, I964), the term for maternal effects is ambiguous in that it is estimated from the average difference between the maternal and paternal arrays. Changing the model to replace it by a paternal effect would not lead to any alteration in the analysis. It is possible, however, to estimate from variance components what proportion of the differential effect is maternal rather than paternal. If the differential effect is entirely maternal, the expectation of the row mean square is $\sigma^{2}+p \sigma^{2} g+p \sigma^{2} m$ and that of the column mean square is $\sigma^{2}+p \sigma^{2} g$, whilst if it is entirely paternal, the 


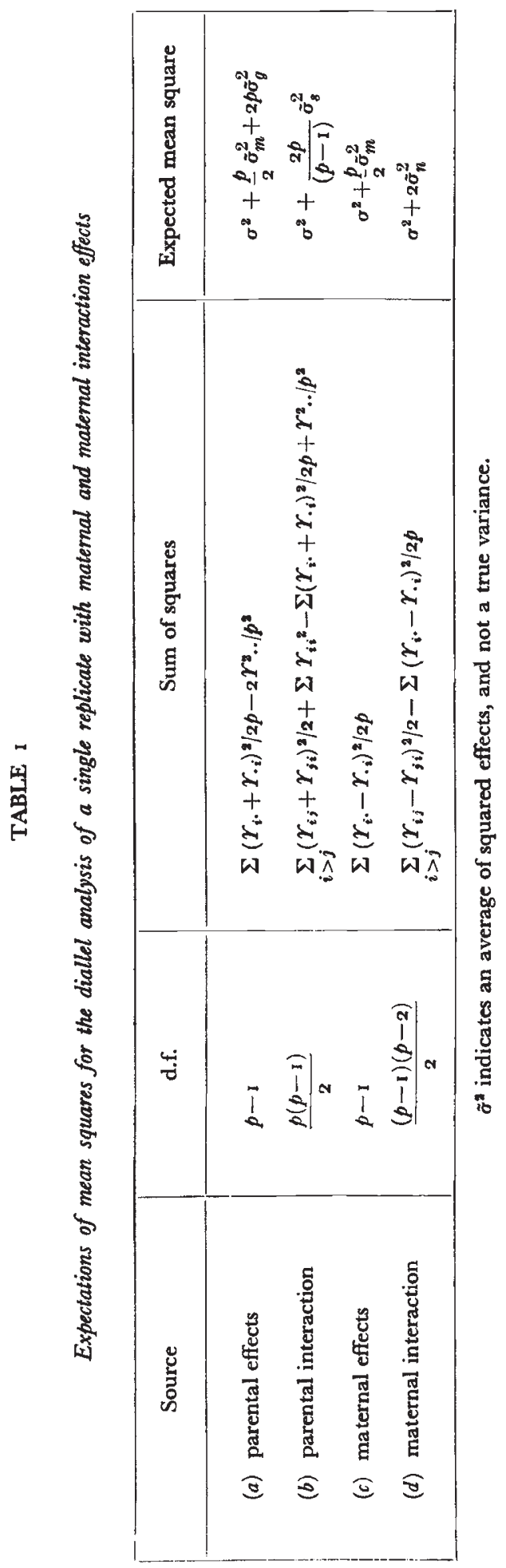


expectations are reversed. Using the estimated values of $\sigma^{2}$ and $\sigma^{2} g$ from the Hayman analysis $\sigma^{2} m$ can be estimated from both the row and the column mean square and the ratio of the estimate from the rows to the sum of the estimates from the rows and the columns will give the relative importance of maternal influence in the differential effects.

$$
\text { Maternal influence }=\frac{\sigma_{m(\text { (row) }}^{2}}{\sigma_{m(\text { row })}^{2}+\sigma_{m(\text { column })}^{2}} .
$$

The procedure will be illustrated with reference to data to be discussed in section 5 , on mean seed size after diallel crosses between five raspberry varieties (D. L. Jennings, to be published) (table 2). It will be

TABLE 2

Raspberry diallel: average seed size and diallel analysis

Mean seed size

\begin{tabular}{|c|c|c|c|c|c|c|}
\hline Female Male & Burnetholm & $511 / 12$ & AR.I & M. Jewel & M. $69 / 139$ & Average \\
\hline $\begin{array}{l}\text { Burnetholm } \\
5 \mathrm{rl} / 12 \\
\text { AR.I } \\
\text { M. Jewel } \\
\text { M. 69/139 } \\
\text { Average }\end{array}$ & $\begin{array}{l}2911 \cdot 2 \\
4135 \cdot 1 \\
3717 \cdot 0 \\
2980 \cdot 7 \\
2519 \cdot 3 \\
3252 \cdot 6\end{array}$ & $\begin{array}{l}3059 \cdot 7 \\
3784^{\cdot 2} \\
3227^{\circ} 0 \\
2677^{\circ} \cdot 6 \\
2655^{\circ} 9 \\
3079^{\circ} 7\end{array}$ & $\begin{array}{l}3401 \cdot 4 \\
4011 \cdot 9 \\
3092 \cdot 5 \\
2844 \cdot 7 \\
2680 \cdot 3 \\
3206 \cdot 1\end{array}$ & $\begin{array}{l}3350 \cdot 9 \\
348 \cdot \cdot 9 \\
3293 \cdot 3 \\
2662 \cdot 3 \\
2860 \cdot 2 \\
3130 \cdot 5\end{array}$ & $\begin{array}{l}3688 \cdot 1 \\
3740 \cdot 8 \\
3441 \cdot 1 \\
2662 \cdot 7 \\
2541 \cdot 5 \\
3214 \cdot 8\end{array}$ & $\begin{array}{l}3282 \cdot 3 \\
3831 \cdot 5 \\
3354 \cdot 2 \\
2764 \cdot 4 \\
2651 \cdot 4 \\
3176 \cdot 8\end{array}$ \\
\hline
\end{tabular}

Seed length $\times$ breadth in eye piece micrometer scale divisions.

Diallel analysis

\begin{tabular}{|c|c|c|c|}
\hline Source of variance & d.f. & Mean square & $F$ test \\
\hline $\begin{array}{l}\text { (a) Parental effect } \\
\text { (b) Genic interaction (s.c.a.) } \\
\text { (c) Maternal effect } \\
\text { (d) Maternal interaction } \\
\text { (c) Residual } \\
\text { (f) Rows } \\
\text { (g) Columns }\end{array}$ & $\begin{array}{r}4 \\
10 \\
4 \\
6 \\
475 \\
4 \\
4 \\
4\end{array}$ & $\begin{array}{r}10430556 \cdot 7 \\
986372 \cdot 1 \\
12994252 \cdot 6 \\
1394127 \cdot 0 \\
131437 \cdot 0 \\
22933819 \cdot 0 \\
490988 \cdot 6\end{array}$ & $\begin{array}{l}\text { a/c } 0.80 \text { not significant } \\
\text { b/e } 7.50 \text { very significant } \\
\text { cle } 98.86 \text { very significant } \\
\text { d/e 10.6 I very significant }\end{array}$ \\
\hline
\end{tabular}

noticed that the parental or genic effect is not significant and that equating the mean squares to their expected values will give a negative value for $\sigma_{g}^{2}$. So we may assume that there is no parental effect and estimate the maternal influence as $0 \cdot 98$. This leads one to suppose that all the average line effects for seed size can be attributed to the influence of the seed parent. The pollen parent, however, does affect seed size, as we see from the highly significant maternal interaction term, but not in any systematic way. 
MATERNAL INTERACTION EFFECTS

669

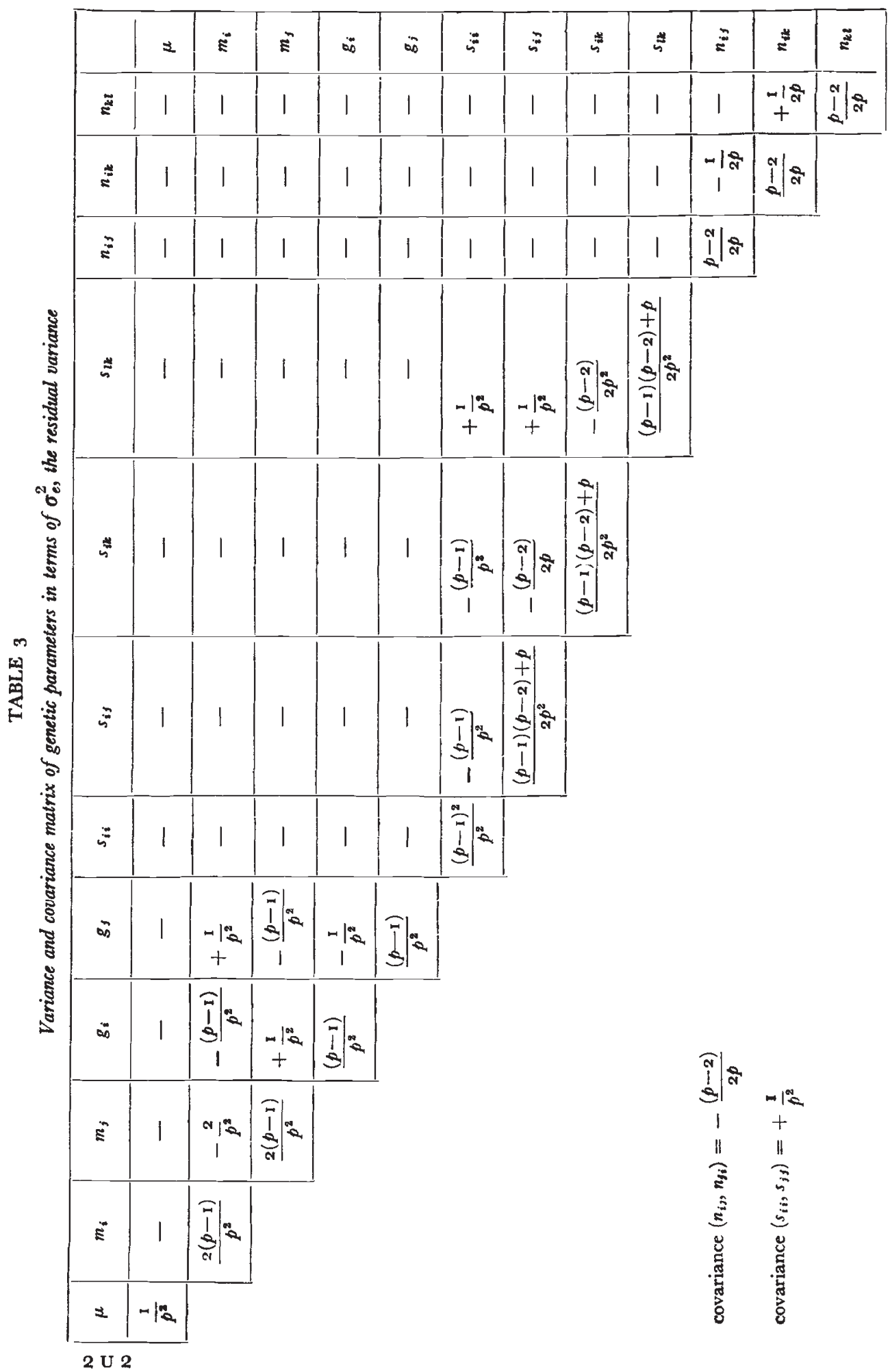




\section{THE PARAMETERS}

Once the significance of each source of variation has been tested, calculating the different parameters for each variety reveals the contribution of each variety to each source.

The equations for estimating the parameters are:

$$
\begin{aligned}
\mu & =Y_{. \cdot / p^{2}} \\
m_{i} & =\left(Y_{i}-Y_{\cdot i}\right) / p \\
g_{i} & =\left(Y_{i \cdot}+Y_{\bullet_{i}}\right) / 2 p-m_{i} / 2-\mu \\
s_{i j} & =\left(Y_{i j}+Y_{j i}\right) / 2-g_{i}-g_{j}-m_{i} / 2-m_{j} / 2-\mu \\
s_{i i} & =Y_{i i}-2 g_{i}-m_{i}-\mu \\
n_{i j} & =r_{i j}-s_{i j}-g_{i}-g_{j}-m_{i}-\mu
\end{aligned}
$$

and their variances and covariances are shown in table 3. The cross totals in these equations and the variances and covariances must all be divided by the number of replicates.

The reasoning used in deriving the expectations is confirmed by the fact that when the $n_{i j} s$ are estimated from equation 6 they do in fact obey the postulated restraints. The covariances arise from the closed nature of the design and from the presence of restraints. They are similar to those given by Federer (1955) for a $p \times q$ factorial in a randomised complete block, but not identical since the parameters fitted also differ. Taking account of relevant covariances, comparisons may be made between values in the same or different sets. If any of the terms in the Hayman analysis are not significant, the values of that set of parameters will not in general reach significance; but if it is decided not to estimate them, the variances and covariances of the remaining sets of parameters will usually be affected. As an example, table 4 shows the variances and covariances for the case in which only the mean, maternal effects and the two interactions are fitted, and it can be seen that the interaction variances and covariances are markedly different from those in table 3 .

\section{DIALLEL ANALYSIS OF RASPBERRY SEED SIZE}

The diallel analysis and estimated parameters for seed size after diallel crosses between 5 raspberry varieties are presented in tables 2 and 5. The data, from a study of seed and germination, refer to seed immediately resulting from diallel pollinations, so that there is no replication over blocks and the residual "within crosses" results from a hierarchical classification. For each cross seed from a number of inflorescences was bulked and a sample taken. Although the parental effect is not significant, the maternal effect is very important, which is not surprising since the testa is a maternal tissue. Genic interactions are also significant, implying that the vigour of embryo and endosperm helps to determine seed size, whilst the significant $(d)$ term implies that interactions between particular seed and pollen parents are also 


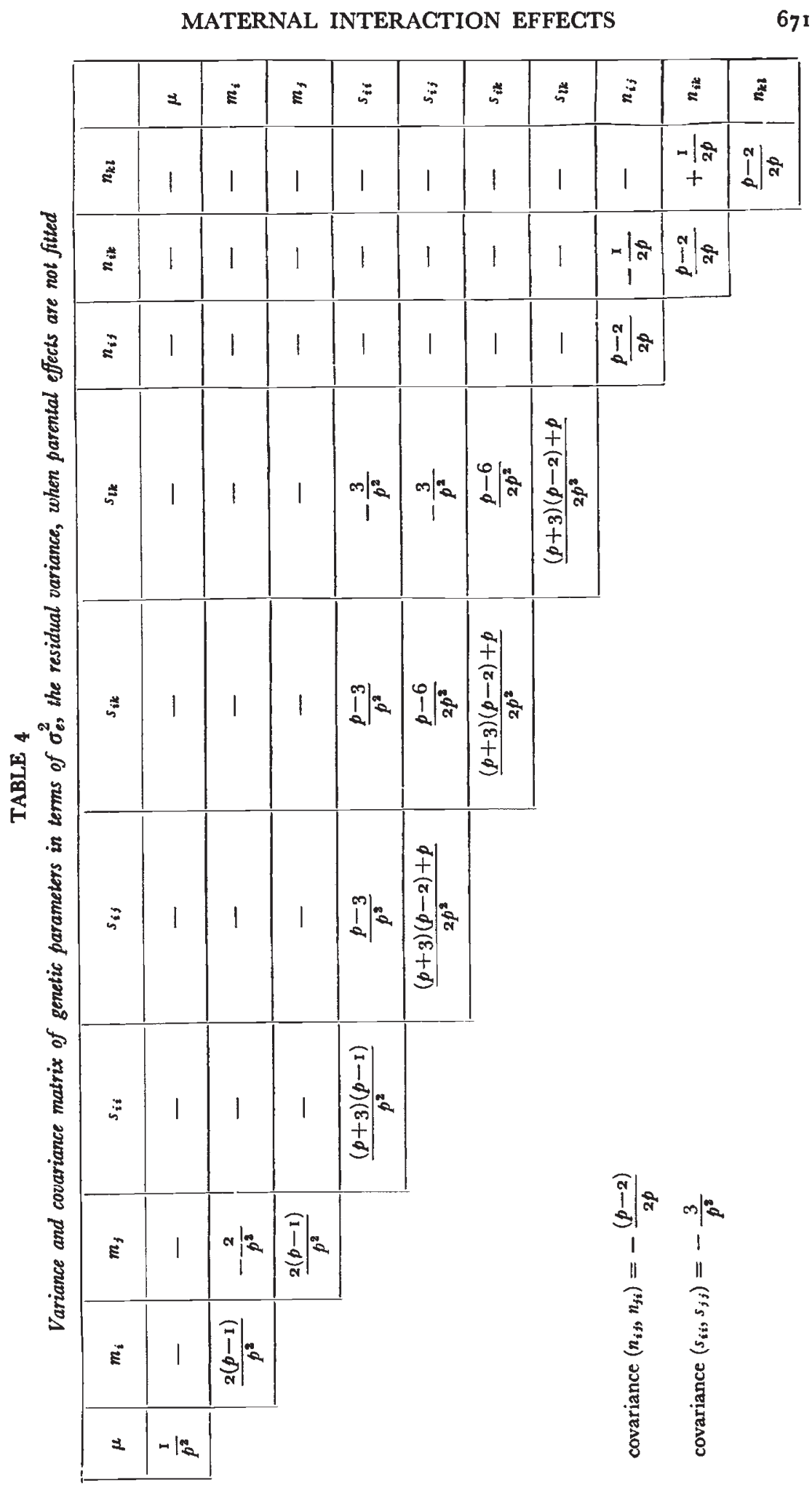




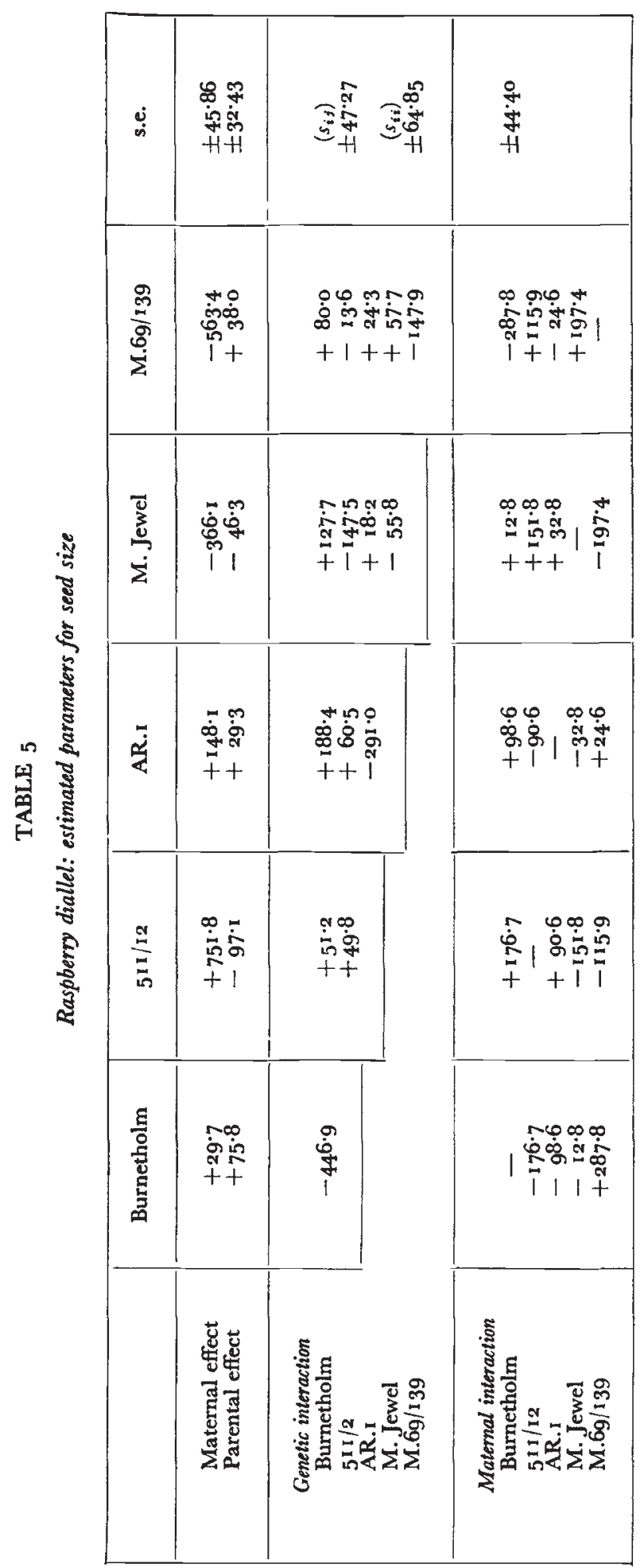


involved. Table 5 shows the size of the various effects for each variety or combination. It is interesting that the estimated value for genetic interaction in the selfs is generally negative, which suggests an early expression or inbreeding depression in this predominantly outbreeding, heterozygous species. This supports the assignment of the self interaction terms to genic interaction but it must be remembered that each will include an unknown component due to maternal interaction. In general the approach does not preclude further division of Hayman's (b) term, but this is of doubtful value where the $(d)$ term is significant.

No assumptions are made about the underlying biological mechanisms and their effects on seed size. The variation is merely divided into four categories, the significance of each ist ested and the size of the effect is estimated; the estimates are valid whatever combinaion of nuclear, cytoplasmic, or physiological interplay between the parents is postulated. In fruit and seed development, work here points to an interaction between the stimulus of the pollen and the response of the maternal tissues, which we visualise in physiological terms as leading to the production of endogenous growth substances in amounts which may or may not be optimal. A difficulty arises in that the " maternal " effect is estimated as the average difference between the female and male arrays and is already an interaction term. With a change of sign it could equally well be considered a male effect, and in this context is probably best considered as the average outcome of the physiological interactions of a variety used as male and as female parent. The regression technique of Durrant ( 1965 ) offers one means of deciding to which parent the effect should be ascribed in the absence of any biological hypothesis, the ratio in section 3 of this paper another.

The physiological stimulus of pollination, though genetically determined, will not be influenced to any extent by genes controlling the early growth of the embryo; for the raspberry seed data the correlation between the parental and maternal effects is not significant $(r=-0.50$, with 3 degrees of freedom; the expected value from table 3 is $-0.707 \mathrm{I}$ ). The situation in which either or both the maternal effect and the maternal interaction are some function of the genic (parental) effect could be further investigated on the lines advocated by Durrant ( 1965 ), to detect a cytoplasmic rather than a physiological interaction.

The importance of the genic effects and their interactions compared to the maternal effects and interactions will vary; in studies of fruit development and metaxenia the physiological aspects will predominate, and be expressed as maternal effects and interactions; in seed and seedling characters both sets of factors may come into play and the genic effects may be modified by the maternal environment to give a large beta component in Durrant's analysis. Characters of the mature plant will normally only show genic effects, unless there is genotrophic plasticity as in flax.

Acknowledgment-I am indebted to Dr D. L. Jennings of this Institute for permission to use his raspberry diallel data. 


\section{REFERENCES}

DURrant, A. 1965. Analysis of reciprocal differences in diallel crosses. Heredity, 20, $573-607$.

FEDERER, W. T. 1955. Experimental Design, New York. p. 214.

HAYMAN, B. I. 1954. The analysis of variance of diallel tables. Biometrics, Io, 235-244.

SPRAGUE, G. F., AND TATUM, L. A. 1942. General vs. specific combining ability in single crosses of corn. Am. Soc. Agron., 34, 923-932.

WEARDEN, s. 1964. Alternative analyses of the diallel cross. Heredity, 19, 669-680. 